\title{
A parametric symmetry breaking transducer
}

\section{Journal Article}

Author(s):

Eichler, Alexander; Heugel, Toni L.; Leuch, Anina; Degen, Christian L.; Chitra, Ramasubramanian; Zilberberg, Oded

Publication date:

2018-06

Permanent link:

https://doi.org/10.3929/ethz-b-000271720

Rights / license:

In Copyright - Non-Commercial Use Permitted

Originally published in:

Applied Physics Letters 112(23), https://doi.org/10.1063/1.5031058

Funding acknowledgement:

177198 - Zeptonewton force sensing on a membrane resonator platform (SNF) 


\title{
A parametric symmetry breaking transducer
}

\author{
Alexander Eichler, ${ }^{1}$ Toni L. Heugel, ${ }^{2}$ Anina Leuch, ${ }^{1,2}$ Christian L. Degen, ${ }^{1}$ R. Chitra, ${ }^{2}$ \\ and Oded Zilberberg ${ }^{2}$ \\ ${ }_{1}^{1}$ Institute for Solid State Physics, ETH Zurich, 8093 Zurich, Switzerland \\ ${ }^{2}$ Institute for Theoretical Physics, ETH Zurich, 8093 Zurich, Switzerland
}

(Received 28 March 2018; accepted 25 May 2018; published online 6 June 2018)

\begin{abstract}
Force detectors rely on resonators to transduce forces into a readable signal. Usually, these resonators operate in the linear regime and their signal appears amidst a competing background comprising thermal or quantum fluctuations as well as readout noise. Here, we demonstrate a parametric symmetry breaking transduction method that leads to a robust nonlinear force detection in the presence of noise. The force signal is encoded in the frequency at which the system jumps between two phase states which are inherently protected against phase noise. Consequently, the transduction effectively decouples from readout noise channels. For a controlled demonstration of the method, we experiment with a macroscopic doubly clamped string. Our method provides a promising paradigm for high-precision force detection. Published by AIP Publishing.

https://doi.org/10.1063/1.5031058
\end{abstract}

Resonators are widely used for the detection and amplification of oscillating signals. In its most basic and ubiquitous form, resonant detection measures the amplitude of oscillation in response to a signal. Examples of resonatorbased sensors range from radar antennas ${ }^{1}$ and nuclear magnetic resonance ${ }^{2}$ to optical antennas, ${ }^{3}$ to gravitational wave detection, ${ }^{4,5}$ and to nanomechanical force transducers. ${ }^{6-9}$ An attractive feature of resonant detection is the possibility of phase-sensitive signal transduction, which can be used to reject unwanted or incoherent signal sources in a lock-in type measurement. In magnetic resonance force microscopy (MRFM), for instance, a small magnetic force acting on a force transducer (a cantilever) is modulated at the transducer's resonance frequency and drives coherent oscillations. ${ }^{10-12}$ The controlled phase and frequency of the force modulation allow us to distinguish weak force signals against an overwhelming noise background.

The sensitivity of a linear amplitude detector is limited by intrinsic fluctuations and by readout noise, both of which can obscure the true response to the force signal. Intrinsic fluctuations include amplitude and phase noise of the resonator vibrations and can stem from many sources. In the particular case of a classical mechanical force transducer, the lowest limit of intrinsic fluctuations is set by the white thermomechanical force noise. This threshold can be decreased by designing resonators with small masses and high mechanical quality factors. ${ }^{13-15}$ Readout noise, on the other hand, is added in the signal amplification process and is typically more pronounced when the resonator vibrations are small. Therefore, as force sensors are scaled down, they usually experience a decrease in intrinsic fluctuations as well as an increase in readout noise. This tradeoff establishes a lower boundary for the forces that can be detected. Pushing this boundary is vital for all sensing techniques.

Standard parametric amplification can reduce readout noise by amplifying the resonator's motion using purely reactive components. Examples of its application include (i) varactor amplifiers used for radio signals, ${ }^{16,17}$ (ii) superconducting parametric amplifiers that have demonstrated readout noise close to that imposed by the laws of quantum mechanics, ${ }^{18-20}$ (iii) squeezed mechanical vibrations, ${ }^{21-24}$ as well as proposals for improved sensitivity of gravitational wave detection. ${ }^{25}$ Such techniques, however, are bound to operate in a regime of relatively small vibrations, i.e., well below the parametric instability threshold. ${ }^{26}$

In this work, we experimentally demonstrate a complementary approach for sensitive force detection that operates beyond the instability threshold of a nonlinear resonator. ${ }^{26,27}$ Our method is based on the distinct double-hysteresis that can be observed in the frequency sweep of a parametric resonator, see Figs. 1(a)-1(c). This double hysteresis arises because the weak applied force breaks the symmetry between the two phase states of the resonator. ${ }^{28}$ Our method is therefore a type of parametric symmetry breaking transducer (PSBT). In contrast to earlier work by Karabalin et $a l .{ }^{29}$ that makes use of symmetry breaking, our technique does not encode the measured force in the coupling strength between two different resonators, but in the shifted bifurcation frequency of a single resonator. ${ }^{28}$ Importantly, even though the resonator vibrations are inherently nonlinear, we show that the transducer has an approximately linear gain characteristic. In comparison with linear amplitude-detection transducers, the PSBT performance degrades faster in the presence of large intrinsic fluctuations. However, it is highly insensitive to readout noise, which makes it, for example, promising for applications with nanomechanical force sensors.

The PSBT can be realized with any system that fulfills the following equation:

$$
\begin{gathered}
\ddot{v}+\omega_{0}^{2}[1-\lambda \cos (2 \omega t)] v+\Gamma \dot{v}+\alpha v^{3} \\
=C \cdot V_{d} \cos (\omega t+\phi)+C \cdot \xi(t) .
\end{gathered}
$$

Here, we have chosen a representation in electrical units to emphasize the generality of the physics involved, i.e., $v=v(t)$ is the measured voltage that is roughly proportional to the resonator displacement. Dots denote differentiation with respect to time $t, \omega$ is a frequency close to the angular 
(a)

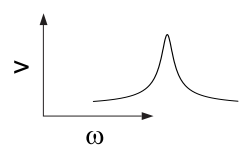

(d)

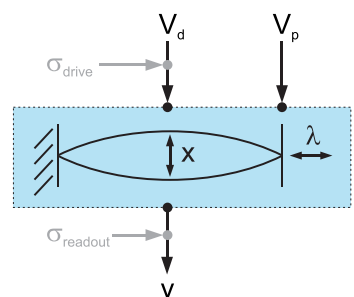

(g)

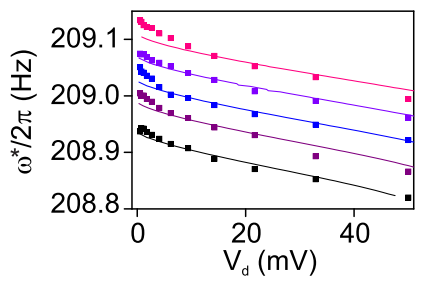

(b)



(e)

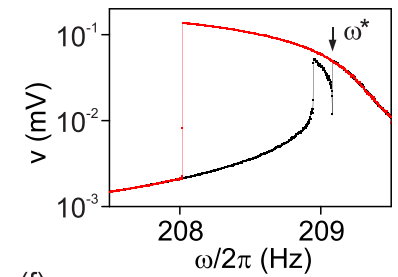

$(\mathrm{f})$

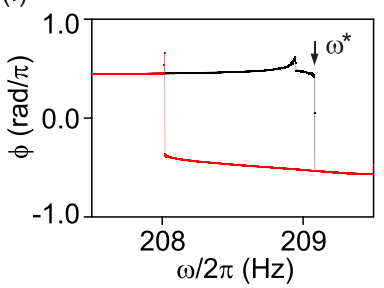

FIG. 1. (a)-(c) Schematic working principle of the PSBT. Response of the resonator to a small force (a) or to a large parametric drive (b), individually. When combined, the two driving sources produce a complex response that has a characteristic second jump (c). Grey and black lines demonstrate how the jump frequency changes as a function of the applied force. (d) Sketch of the setup showing input voltages $V_{d}$ and $V_{p}$, as well as the measured output voltage $v$ (which corresponds to a mechanical vibration $x$ ). Added voltage noise sources are indicated by their standard deviations $\sigma_{\text {drive }}$ and $\sigma_{\text {readout }}$. (e) and (f) Amplitude and phase of a frequency sweep with parametric drive and external drive applied simultaneously, with $V_{p}=0.25 \mathrm{~V}$ and $V_{d}=0.1 \mathrm{~V}$. Red and black correspond to sweeps with decreasing and increasing frequencies, respectively. The arrow marks the frequency $\omega^{*}$ at which the PSBT jump occurs. (g) Position of frequency jump, $\omega^{*}$, as a function of applied voltage, $V_{d}$. The curves were offset for better visibility and correspond to $V_{p}=0.21 \mathrm{~V}-0.25 \mathrm{~V}$ in steps of $0.01 \mathrm{~V}$ (bottom to top). Lines denote theory fits using $\alpha=-6.7 \times 10^{6} \mathrm{~V}^{-2} \mathrm{~s}^{-2}$ as the only fitting parameter. Slow frequency drifts occurring between sets were compensated.

eigenfrequency $\omega_{0}$ of the resonator, $\Gamma$ is a linear damping constant, and $\alpha$ represents a nonlinear (Duffing) spring constant. $V_{d}$ is the amplitude of an applied external driving voltage that is proportional to a force applied to the resonator with phase $\phi$, and $C$ is a gain factor (See supplementary material). $\xi(t)$ is an additive intrinsic fluctuating drive with standard deviation $\sigma_{\text {drive }}$. In addition to the external drive, we also modulate the resonance frequency at a rate $2 \omega$ and with a modulation depth $\lambda$, which we refer to as "parametric drive" and which we control with a voltage signal of amplitude $V_{p}$. Beyond a threshold value $V_{t h} \propto \lambda_{t h}=2 \Gamma / \omega_{0}$, this excitation leads to large and stable oscillations of the resonator. In our system, there is an additional nonlinear damping term in Eq. (1), $\eta v^{2} \dot{v}$, that has a negligible influence on the PSBT performance.

Our experimental demonstration is based on a macroscopic doubly clamped string that vibrates mechanically in accord with Eq. (1), see Fig. 1(d) ${ }^{28}$ [see supplementary material for a derivation of Eq. (1) for a mechanical resonator]. We characterize the resonator at both small and large vibration $v \propto x$. With a small external force applied and with $\lambda=0$, the resonator behaves linearly [Fig. 1(a)], which allows us to extract $\omega_{0} /(2 \pi) \sim 208.8 \mathrm{~Hz}, \Gamma=\omega_{0} / Q$ with quality factor $Q=2150$, and $C=430 \mathrm{~s}^{-2}$. To fit the nonlinear constants $\alpha$ and $\eta$, we

set $V_{d}=0$ and drive the system to large amplitudes with $V_{p}>V_{t h}=80 \mathrm{mV}$. From a fit to the nonlinear response [Fig. 1(b)], we obtain $\alpha / \eta=1875 \mathrm{~s}^{-1}$ (See supplementary material).

To perform force measurements, we exploit the doublehysteresis pattern that emerges when parametric and external drives are applied simultaneously [Fig. 1(e)]. The underlying physics is governed by a symmetry breaking in the parametric phase states. ${ }^{28,30-32}$ The second jump of the upsweep at $\omega^{*}$ is a direct consequence of the interplay between the two drives. In the absence of noise, the jump frequency is expected to depend approximately linearly on $V_{d}$ for a range of forces. In the following, we shall focus on the accompanying phase jump at $\omega^{*}$, which corresponds roughly to $\pi$ radians even when the jump is small in amplitude [Fig. 1(f)]. The phase jump is the most convenient experimental signature for our force detection method.

In Fig. 1(g), we experimentally demonstrate the relationship between $\omega^{*}$ and $V_{d}$ for various values of the parametric modulation depth. The corresponding theoretical results are obtained by studying the time-averaged slow dynamics of the system and the jump frequency $\omega^{*}$ is derived using a bifurcation analysis of the equations of motion (See supplementary material). The almost-linear dependence of $\omega^{*}$ on $V_{d}$ indicates that usage of the calibrated force sensor is straightforward in spite of the complex nonlinear physics involved.

We now evaluate the performance of our method in the presence of noise. Since our resonator operates far above any natural noise levels, we artificially add white voltage noise either in the form of intrinsic fluctuations $\xi(t)$ or as a fluctuating component of the measured voltage $v$, with standard deviations $\sigma_{\text {drive }}$ and $\sigma_{\text {readout }}$, respectively [see Fig. 1(d)]. For comparison, we first use the resonator as a simple linear transducer without parametric drive. In Figs. 2(a) and 2(c), we show that the resulting amplitude signal in the presence of either of the noise channels is almost entirely obscured by the fluctuations. Next, we repeat the sweep with added parametric drive to operate the resonator as a PSBT. Even though
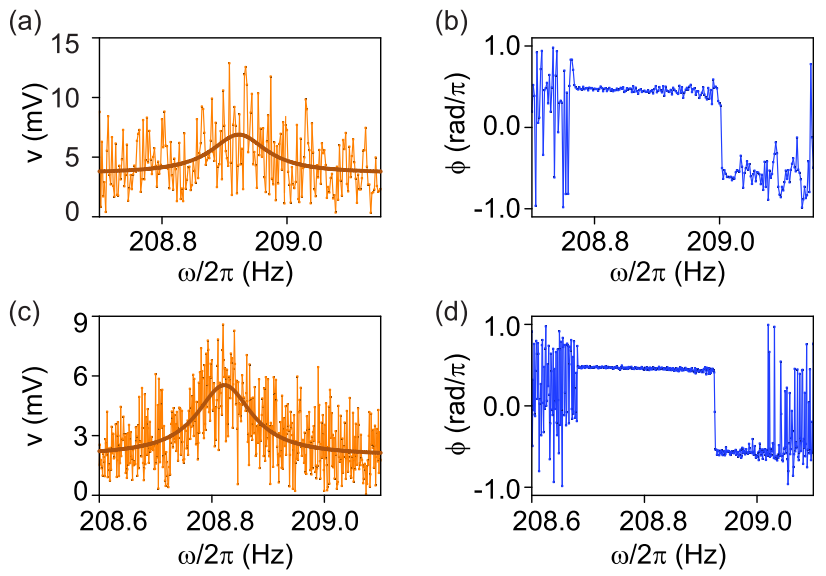

FIG. 2. (a) Linear sensor $\left(V_{p}=0\right)$ with artificial readout noise (standard deviation $\sigma_{\text {readout }}=1.8 \mathrm{mV}$ within the measurement bandwidth). A voltage with amplitude $V_{d}=10 \mathrm{mV}$ was swept in frequency and the response detected with a lock-in amplifier. The total sweep time was 1920 s. (b) The same sweep performed in the PSBT mode $\left(V_{p}=0.25 \mathrm{~V}\right)$. Here, we detect the phase rather than the amplitude. (c) Linear sensor with artificial intrinsic noise power spectral density $S_{\text {drive }}=2.96 \times 10^{-4} \mathrm{~V}^{2} / \mathrm{Hz}$ and $V_{d}=10 \mathrm{mV}$. The total sweep time was $4080 \mathrm{~s}$. (d) The same sweep performed in the PSBT mode $\left(V_{p}=0.25 \mathrm{~V}\right)$. Solid lines in (a) and (c) are Lorentzian fits. 
the fluctuations in the phase are noticeable in the signal, the phase jump stands out clearly in Figs. 2(b) and 2(d).

Comparing the linear transducer and the PSBT, we have shown that the latter exhibits a surprising robustness to both intrinsic and readout noise. However, this does not yet characterize the precision of the PSBT in estimating the amplitude of the external signal $V_{d}$ from the jump frequency $\omega^{*}$, namely, we need to know the variance of the PSBT estimation. To this end, we numerically simulate repeated measurements of $V_{d}$ using both the linear and PSBT methods. The simulations enable us to obtain large statistics of the detection performance in the presence of controllable and independent noise channels. In Figs. 3(a) and 2(b), exemplary histograms of the estimated signal are presented for readout and intrinsic noise channels, respectively. The PSBT histograms exhibit an almost-Gaussian distribution whose standard deviation quantifies a standard error $\Delta$ (See supplementary material) for the force measurement.

In Figs. 3(c) and 3(d), we show how noise influences $\Delta$. We systematically observe that the impact of intrinsic noise on the PSBT is larger than its effect on the corresponding linear transducer. The intrinsic fluctuations increase the chance for the PSBT to flip prematurely, which translates into frequency noise in the estimation. However, for readout noise, the situation is manifestly opposite and the PSBT significantly outperforms the linear transducer. This is a direct consequence of the fact that the PSBT signal is encoded in the phase of the oscillation, while the phase noise is reduced by driving the oscillator to a large amplitude. The PSBT, thus, effectively decouples from the readout noise channel. Our analysis indicates that the PSBT will have a better signal-to-noise ratio in situations where the detection is limited by readout noise.

Finally, we would like to discuss some limitations of the PSBT scheme: (i) the PSBT relies on a joint sweep of the
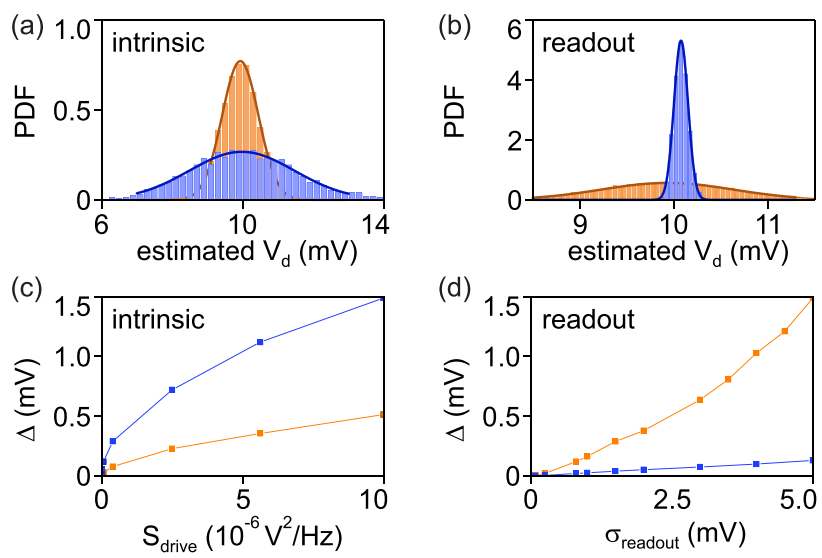

FIG. 3. (a) and (b) Probability density function (PDF) of the driving voltage $V_{d}$ estimated from simulated sweeps in the presence of intrinsic noise and readout noise, respectively. Orange bars denote results obtained with the linear sensor, and blue bars are for the PSBT sensor (note corresponding colors in Fig. 2). Solid lines correspond to Gaussian fits that allow extracting the standard error $(\Delta)$. All resonator parameters were matched to the experimental device. The intrinsic noise in (a) has a power spectral density of $S_{\text {drive }}$ $=10^{-5} \mathrm{~V}^{2} / \mathrm{Hz}$ and the readout noise in (b) has a standard deviation of $3 \mathrm{mV}$. In each case, we simulated 3000 sweeps with intrinsic noise and 10000 sweeps with readout noise. (c) and (d) Standard error of estimated $V_{d}$ as a function of the standard deviation of applied intrinsic and readout noise, respectively. frequencies of both external and parametric drives. This implies that the measured force can be modulated at a desired frequency and with a controlled phase, similar to MRFM; ${ }^{10}$ (ii) the dynamic range, i.e., the range of forces that can be measured, depends on the parametric drive. When the resonator amplitude in response to the measured force becomes comparable to that of the parametric oscillation, the double hysteresis is replaced by a qualitatively different behavior and the PSBT scheme breaks down. In our experiment and for $V_{d}=0.25 \mathrm{~V}$, this resulted in an upper limit of $V_{d} \approx 0.15$ $\mathrm{V}$; (iii) the PSBT method is sensitive to frequency noise. Fluctuations of $\omega_{0}$ will lead to shifts in $\omega^{*}$ and distort the estimation of the measured force; (iv) the bandwidth (i.e., the repetition rate) of force measurements with our method is given by the sweeping speed and therefore ultimately by the resonator's quality factor. (v) Systematic errors in the force estimation can arise with the PSBT due to premature switching, delayed resonator response, ${ }^{33,34}$ or phase noise bias. In the situations we studied, these systematic errors can be removed through proper calibration (See supplementary material).

We have demonstrated that the PSBT has several attractive features that set it apart from a linear force transducer. The PSBT makes use of parametric phase states, which are intrinsically protected against amplitude and phase noise. Since the measured force is extracted from a frequency as opposed to amplitude, the PSBT can measure small forces even while operating at relatively large oscillation amplitudes. This feature makes the PSBT highly tolerant to readout noise similar to frequency-modulated, feedback-driven oscillators. ${ }^{35}$ However, in contrast to the slow frequency modulation rate used in the latter, our method can detect forces at frequencies close to the eigenfrequency of the transducer itself. We believe that the PSBT is promising for force detection experiments with nanomechanical resonators such as carbon nanotubes or graphene, as well as for the detection of electrical signals with Josephson parametric resonators. Further work will focus on the performance of PSBT sensors in the quantum realm.

See supplementary material for a derivation of Eq. (1), a characterization of the experimental system, an explanation regarding the bifurcation analysis used to theoretically determine the jump frequency, as well as details on the numerical simulations presented in Fig. 3.

We acknowledge fruitful discussions with L. Papariello and technical support from C. Keck, P. Märki, M. Baer, and the mechanical workshop team of the Department of Physics at ETH Zurich. This work received financial support from the Swiss National Science Foundation (No. CRSII5_177198/1).

${ }^{1}$ M. I. Skolnik, Introduction to Radar Systems (McGraw Hill Book Co., 2000). ${ }^{2}$ I. I. Rabi, J. R. Zacharias, S. Millman, and P. Kusch, Phys. Rev. 53, 318 (1938).

${ }^{3}$ L. Novotny and N. van Hulst, Nat. Photonics 5, 83 (2011).

${ }^{4}$ J. Weber, Phys. Rev. 117, 306 (1960).

${ }^{5}$ B. P. Abbott, R. Abbott, T. D. Abbott, M. R. Abernathy, F. Acernese, K. Ackley, C. Adams, T. Adams, P. Addesso, R. X. Adhikari et al. (LIGO Scientific Collaboration and Virgo Collaboration. ), Phys. Rev. Lett. 116, 061102 (2016).

${ }^{6}$ G. Binnig, C. F. Quate, and C. Gerber, Phys. Rev. Lett. 56, 930 (1986). 
${ }^{7}$ D. Rugar, H. J. Mamin, P. Guethner, S. E. Lambert, J. E. Stern, I. McFadyen, and T. Yogi, J. Appl. Phys. 68, 1169 (1990).

${ }^{8}$ H. J. Mamin and D. Rugar, Appl. Phys. Lett. 79, 3358 (2001).

${ }^{9}$ J. L. Arlett, J. R. Maloney, B. Gudlewski, M. Muluneh, and M. L. Roukes, Nano Lett. 6, 1000 (2006).

${ }^{10}$ J. A. Sidles, Appl. Phys. Lett. 58, 2854 (1991).

${ }^{11}$ D. Rugar, R. Budakian, H. J. Mamin, and B. W. Chui, Nature 430, 329 (2004).

${ }^{12}$ M. Poggio and C. L. Degen, Nanotechnology 21, 342001 (2010).

${ }^{13}$ J. Moser, J. Güttinger, A. Eichler, M. J. Esplandiu, D. E. Liu, M. I. Dykman, and A. Bachtold, Nat. Nanotechnol. 8, 493 (2013).

${ }^{14}$ Y. Tsaturyan, A. Barg, E. S. Polzik, and A. Schliesser, Nat. Nanotechnol. 12, 776 (2017).

${ }^{15}$ M. Héritier, A. Eichler, Y. Pan, U. Grob, I. Shorubalko, M. D. Krass, Y. Tao, and C. L. Degen, Nano Lett. 18(3), 1814-1818 (2018).

${ }^{16}$ H. Heffner and G. Wade, J. Appl. Phys. 29, 1321 (1958).

${ }^{17} \mathrm{P}$. Penfield and R. P. Rafuse, Varactor Applications (MIT Press, Cambridge, MA, 1962).

${ }^{18}$ L. Kuzmin, K. Likharev, V. Migulin, and A. Zorin, IEEE Trans. Magn. 19, 618 (1983).

${ }^{19}$ B. Yurke, L. R. Corruccini, P. G. Kaminsky, L. W. Rupp, A. D. Smith, A. H. Silver, R. W. Simon, and E. A. Whittaker, Phys. Rev. A 39, 2519 (1989).

${ }^{20}$ A. Roy and M. Devoret, "Quantum microwaves/micro-ondes quantiques," C. R. Phys. 17, 740 (2016).
${ }^{21}$ D. Rugar and P. Grütter, Phys. Rev. Lett. 67, 699 (1991).

${ }^{22}$ A. Szorkovszky, A. C. Doherty, G. I. Harris, and W. P. Bowen, Phys. Rev. Lett. 107, 213603 (2011).

${ }^{23}$ M. Poot, K. Y. Fong, and H. X. Tang, Phys. Rev. A 90, 063809 (2014).

${ }^{24}$ I. Mahboob, H. Okamoto, K. Onomitsu, and H. Yamaguchi, Phys. Rev. Lett. 113, 167203 (2014).

${ }^{25}$ C. M. Caves, Phys. Rev. D 23, 1693 (1981).

${ }^{26}$ M. C. Lifshitz and R. Cross, "Nonlinear dynamics of nanomechanical and micromechanical resonators," in Reviews of Nonlinear Dynamics and Complexity (Wiley-VCH, 2009), pp. 1-52.

${ }^{27}$ I. Mahboob and H. Yamaguchi, Nat. Nanotechnol. 3, 275 (2008).

${ }^{28}$ A. Leuch, L. Papariello, O. Zilberberg, C. L. Degen, R. Chitra, and A. Eichler, Phys. Rev. Lett. 117, 214101 (2016).

${ }^{29}$ R. B. Karabalin, R. Lifshitz, M. C. Cross, M. H. Matheny, S. C. Masmanidis, and M. L. Roukes, Phys. Rev. Lett. 106, 094102 (2011).

${ }^{30}$ D. Ryvkine and M. I. Dykman, Phys. Rev. E 74, 061118 (2006).

${ }^{31}$ J. F. Rhoads and S. W. Shaw, Appl. Phys. Lett. 96, 234101 (2010).

${ }^{32}$ L. Papariello, O. Zilberberg, A. Eichler, and R. Chitra, Phys. Rev. E 94, 022201 (2016).

${ }^{33}$ C. van den Broeck and P. Mandel, Phys. Lett. A 122, 36 (1987).

${ }^{34}$ N. G. Stocks, R. Mannella, and P. V. E. McClintock, Phys. Rev. A 40, 5361 (1989).

${ }^{35}$ T. R. Albrecht, P. Grütter, D. Horne, and D. Rugar, J. Appl. Phys. 69, 668 (1991). 